\title{
Acute Disseminated Encephalomyelitis
}

National Cancer Institute

\section{Source}

National Cancer Institute. Acute Disseminated Encephalomyelitis. NCI Thesaurus. Code C34578.

An inflammatory process affecting the central nervous system. It is characterized by perivascular demyelination. Symptoms usually follow an acute viral infection or immunization and include fever, confusion, somnolence and involuntary movements. It may lead to coma and death. 